\title{
Hypertrophic olivary degeneration: The forgotten triangle of Guillain and Mollaret
}

Sir,

Hypertrophic olivary degeneration (HOD), a form of transsynaptic degeneration, results from a lesion of the dento-rubro-olivary pathway (also called the anatomical triangle of Guillain and Mollaret). The degeneration is unique in that it is associated, at least for a period of time, with the hypertrophy rather than atrophy of inferior olivary nucleus. ${ }^{[1]} \mathrm{We}$ present two cases of HOD. The clinical presentation was dentatorubral tremor in both cases, but palatal myoclonus, the hallmark of this disorder, had not yet developed in either.

A 50-year-old man, with a history of old pontine infarct resulting in right-sided facial and trigeminal palsy, presented with progressively worsening upper extremity tremor and cerebellar signs. Neurological examination revealed weakness of right masticatory muscles, facial hemianesthesia and cerebellar signs. Palatal myoclonus was not observed. Magnetic resonance imaging (MRI) of the brain revealed cystic encephalomalacia in the deep dorsal pons in right paraventricular location (the site of motor and sensory nucleus of the trigeminal nerve). In addition, the ipsilateral inferior olivary nucleus showed hyperintense signal on T2/PD images suggestive of HOD. Striking cerebellar cortical atrophy was evident on the contralateral side. The right pterygoid, masetter and temporalis muscles showed denervation atrophy (manifested by hyperintense T2/FLAIR signal) secondary to the initial infarct [Figures 1-4].

A 45-year-old female, with a past history of nonhypertensive right cerebellar hemorrhage documented by a computerized tomography (CT) underwent magnetic resonance imaging (MRI) of brain for recent onset of upper limb tremor. MRI revealed a chronic deep right cerebellar hemorrhage (manifested as hypointensity on T2 weighted images-due to hemosiderin staining); hyperintense signal on T2/PD/FLAIR images, consistent with HOD was seen on opposite side [Figure 5].

The anatomical triangle of Guillian and Mollaret consists of a central tegmental tract connecting red nucleus to inferior olivary nucleus, dentatorubral tract (connecting dentate nucleus to contralateral red nucleus via superior cerebellar peduncle), and inferior cerebellar peduncle connecting inferior olivary nucleus to contralateral cerebellar cortex and dentate nucleus. ${ }^{[1]}$ The dentatorubral 

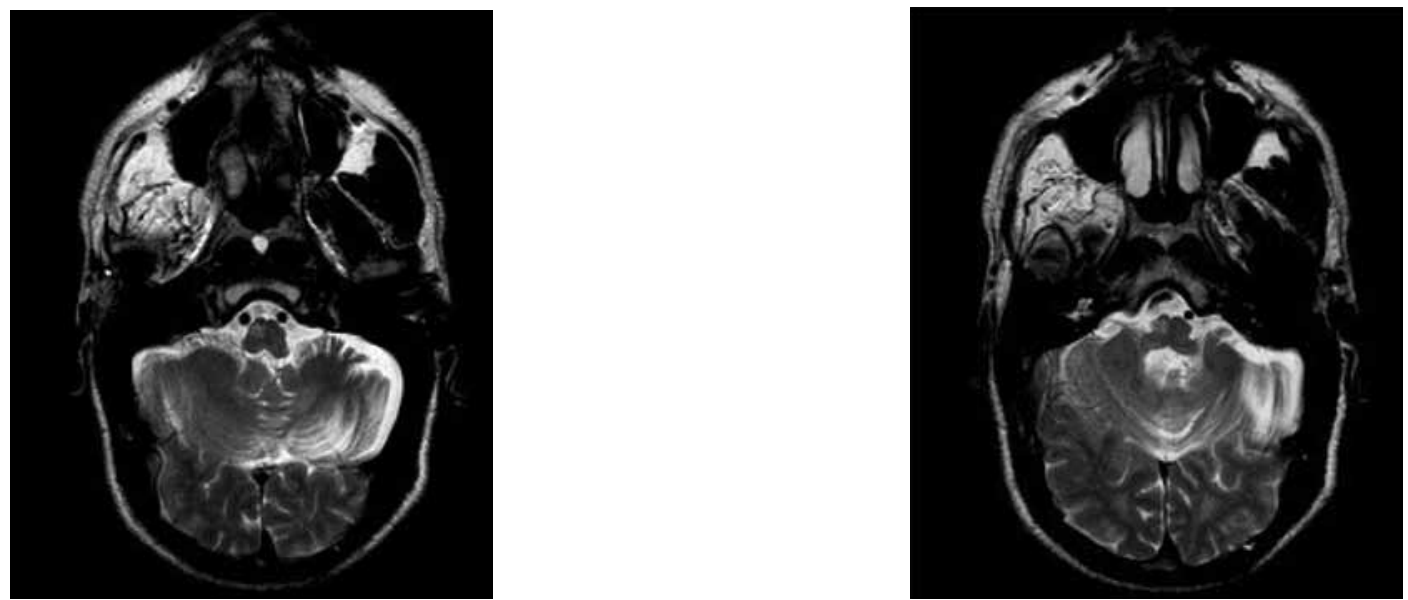

Figures 1 and 2: Hyperintense signal of right inferior olivary nucleus is noted (black arrow) on axial T2 weighted images, suggestive of chronic HOD (the hypertrophy resolves in chronic cases, and only hyperintense signal persists). Hyperintense signal of right massetter and pterygoids is seen (white arrow), which is due to denervation changes secondary to involvement of trigeminal nerve nucleus because of the original infarct. Left cerebellar cortical atrophy is also noted

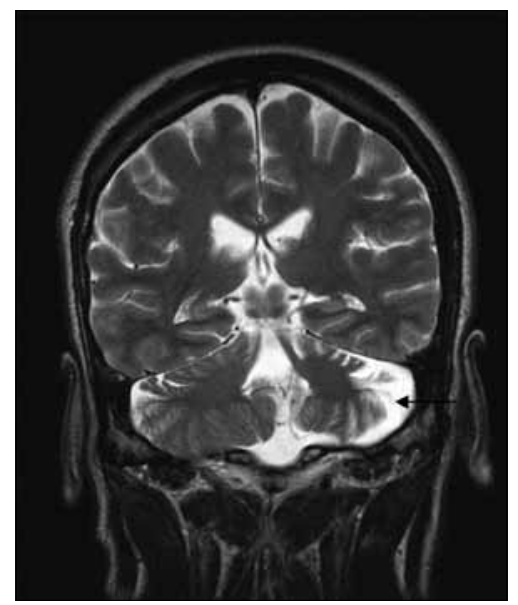

Figure 3: Coronal T2 weighted image showing striking left cerebellar cortical atrophy

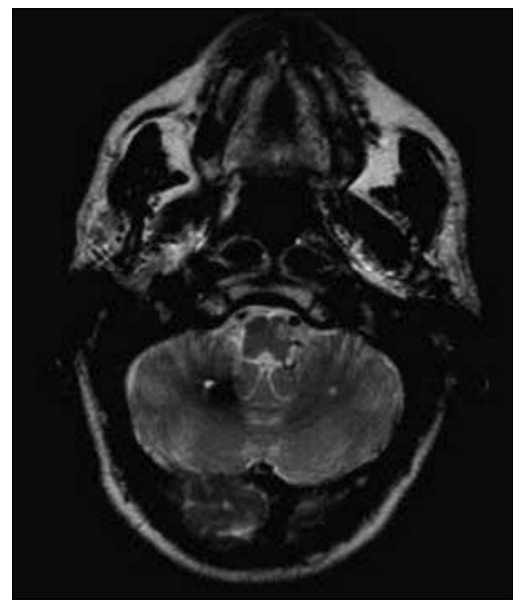

Figure 5: A hypointense lesion seen on axial T2 image is seen in the right deep cerebellar hemisphere suggestive of hemosiderin staining due to old hemorrhage. HOD is seen on the contralateral side

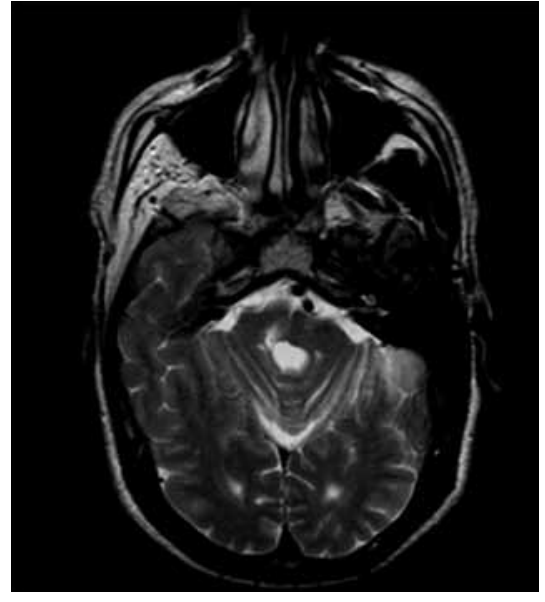

Figure 4: There is an area of hyperintese signal in right paramedian pons that represents an old infarct. The hyperintense signal in the right temporalis is due to the resulting trigeminal palsy

olivary connections were described by Guillian and Mollaret ${ }^{[2]}$ in 1931 as the anatomic connections related to palatal myoclonus, which is a common clinical association. The most common lesions that result in HOD involve the brainstem or pons: The spectrum of lesions varies from ischemic infarction, demyelination, hemorrhage of various etiologies or trauma. ${ }^{[3-5]}$ The olivary hypertrophy appears in a delayed fashion (four to six months) and resolves in three to four years; however, the T2 hyperintense signal and the abnormal movements persist for years. ${ }^{[6-7]}$ When the primary lesion is limited to the central tegmental tract, olivary hypertrophy is ipsilateral. This is illustrated by our first case in which a paramedian pontine infarct led to HOD on the same side. When the primary lesion is in the dentate nucleus or superior cerebellar peduncle, olivary degeneration is contralateral. This is shown in our second 
case in whom a cerebellar hemorrhage due to a cavernous angioma resulted in contralateal olivary changes. When both the central tegmental tract and superior cerebellar peduncle are involved, bilateral involvement results. Pathologically, olivary enlargement is due to vacuolar degeneration of cytoplasm with increased astrocytes; gliosis follows 15-20 months after the onset of primary lesion. ${ }^{[7]}$ The first case showed striking cerebellar cortical atrophy contralateral to the olivary degeneration; this is mediated by the olivodentate fibers which are essential for maintenance of normal cerebellar hemisphere just as the first two limbs of the triangle are essential for health of olivary nucleus.

Various clinical features include palatal myoclonus, dentatorubral tremor and ocular myoclonus. Palatal myoclonus develops 10-11 months after primary lesion, although it may not always be seen (as in both of our cases). ${ }^{[8-9]}$ The various differential diagnoses include demyelination, tumor (astrocytoma), wallerian degeneration (involving the corticospinal tract), infarction and granulomatous pathology. These can be distinguished by clinical and imaging features that include a contrast-enhanced study; most neoplasms and infectious processes would result in contrast enhancement. Wallerian degeneration, amyatrophic lateral sclerosis (ALS) and adrenoleukodystrophy demonstrate hyperintensity of corticospinal tracts and not the inferior olivary nucleus. ${ }^{[1]}$ The most important clue is the presence of remote lesion, and contralateral cerebellar atrophy which makes diagnosis other than HOD impossible. ${ }^{[1]}$

\section{Naseer A. Choh, Suhil A. Choh', Maji Jehangir}

Departments of Radiology and ${ }^{1}$ Pediatrics, SMHS Hospital, Srinagar, India. E-mail: suhilchoh@rediffmail.com

DOI: $10.4103 / 0028-3886.55587$

\section{References}

1. Salamon-Murayama N, Russell EJ, Rabin BM. Diagnosis please. Case 17: hypertrophic olivary degeneration secondary to pontine hemorrhage. Radiology 1999;213:814-7.

2. Kim SJ, Lee JH, Suh DC. Cerebellar MR changes in patients with olivary hypertrophic degeneration.AJNR Am J Neuroradiol 1994;15:1715-9.

3. Goyal M, Versnick E, Tuite P, Cyr JS, Kucharczyk W, Montanera W, Willinsky R, Mikulis D. Hypertrophic olivary degeneration: metaanalysis of the temporal evolution of MR findings. A.JNR Am J Neuroradiol 2000;21:1073-7.

4. Birbamer G, Gerstenbrand F, Aichner F, Buchberger W, Chemelli A, Langmayr J, et al. MR-imaging of post-traumatic olivary hypertrophy. Funct Neurol 1994;9:183-7.

5. Kitajima M, Korogi Y, Shimomura O, Sakamoto Y, Hirai T, Miyayama $\mathrm{H}$, et al. Hypertrophic olivary degeneration: MR imaging and pathologic findings.Radiology 1994;192:539-43.

6. Uchino A, Hasuo K, Uchida K, Matsumoto S, Tsukamoto Y, Ohno M, et al.Olivary degeneration after cerebellar or brain stem haemorrhage: MRI.Neuroradiology 1993;35:335-8.

7. Goto N, Kaneko M. Olivary enlargement: chronological and morphometric analyses.Acta Neuropathol 1981;54:275-82.

8. Matsuo F, Ajax ET. Palatal myoclonus and denervation supersensitivity in the central nervous system.Ann Neurol 1979;5:72-8.

9. Robin JJ, Aleala H. Olivary hypertrophy without palatal myoclonus associated with a metastatic lesion to the pontine tegmentum. Neurology $1975 ; 25: 771-5$.

Accepted on 19-01-2009 